\title{
Expression of Laminin-5- $\gamma$-2 Chain in Intraductal Papillary-Mucinous and Invasive Ductal Tumors of the Pancreas
}

\author{
Noriyoshi Fukushima, M.D., Michiie Sakamoto, M.D., Setsuo Hirohashi, M.D. \\ Clinical Laboratory, National Cancer Center Hospital (NF); and Pathology Division, National Cancer \\ Center Research Institute (MS, SH), Tokyo, Japan
}

The laminin-5- $\gamma$-2 chain is expressed in various invasive carcinoma cells. To clarify the relationship between laminin- 5 expression and the development of intraductal papillary-mucinous tumors (IPMTs), we performed an immunohistochemical study of 26 IPMTs and 30 invasive ductal adenocarcinomas. Cases were classified into five groups: intraductal papillary-mucinous adenoma (Group A; $n=8$ ), adenocarcinoma without invasion (Group B; $n=3$ ), adenocarcinoma with minimal invasion (Group C; $\boldsymbol{n}$ $=5$ ), adenocarcinoma with macroscopically evident invasion (Group D; $n=10$ ), and invasive ductal adenocarcinoma (conventional type; Group E; $\boldsymbol{n}=$ 30 ). In the invasive components of Groups $D$ and $E$, laminin-5 was expressed in $80 \%$ and $100 \%$ of cases, respectively. In the intraductal components of IPMTs, expression of laminin-5 was not seen in Groups A and B, whereas they were seen in one case in Group C (20\%) and in seven in Group D (70\%). Most of the staining patterns of the intraductal components were focal and scattered. Laminin-5$\gamma-2$ expression in the intraductal components of IPMTs tends to increase as tumors develop and may be a indicator of the potential invasiveness of the tumor cells.

KEY WORDS: Immunohistochemistry, Intraductal papillary-mucinous tumor, Laminin-5- $\gamma$-2 chain, Pancreas.

Mod Pathol 2001;14(5):404-409

Copyright @ 2001 by The United States and Canadian Academy of Pathology, Inc

VOL. 14, NO. 5, P. 404, 2001 Printed in the U.S.A.

Date of acceptance: January 4, 2001.

This research was supported in part by a Grant-in-Aid for Second Term Comprehensive 10-year Strategy for Cancer Control from the Ministry of Health and Welfare, Japan.

Address reprint requests to: Setsuo Hirohashi, M.D., Pathology Division, National Cancer Center Research Institute, 1-1 Tsukiji 5-chome, Chuo-ku,

Tokyo 104-0045, Japan; email: shirohas@ncc.go.jp; fax: +81-3-3248-2463.
Intraductal papillary-mucinous tumors (IPMTs) of the pancreas are mucus-producing neoplasms that show intraductal epithelial proliferation with diffuse or segmental dilatation of the main and/or branch pancreatic ducts (1-3). There are various lesions ranging from benign to malignant. Most IPMT cases are adenomas or noninvasive/minimally invasive carcinomas that are resectable and show no recurrence $(4,5)$. However, invasive cases have variable prognoses, depending on the degree of tumor extension. The 3- and 5-year survival rates of patients with invasive IPMT have been reported as $67 \%$ and $28 \%$, respectively (6). It is important to judge tumor invasion precisely.

Laminins are a family of basement membrane proteins that are associated with cell differentiation, adhesion, and migration, as well as being structural components $(7,8)$. Laminin variants are composed of one heavy $\alpha$-chain and lighter $\beta$ - and $\gamma$-chains, which are linked to each other through an $\alpha$-helical coiled structure $(7,8)$. These chains can form a variety of trimeric isoforms, but relatively little is known about their biological roles. The laminin-5- $\gamma$-2 chain is known to be expressed in cells of squamous cell carcinoma and various adenocarcinomas (9-16). According to some reports, the laminin-5- $\gamma$-2 chain is found in front-line invasive cancer cells at the epithelial-stromal interface and plays an important role in cancer cell invasion $(9-16)$.

We investigated the localization of laminin-5- $\gamma$-2 chain protein in IPMTs ranging from adenoma to invasive carcinoma to determine its usefulness in the evaluation of these tumors.

\section{MATERIALS AND METHODS}

\section{Materials}

We collected 26 surgically resected IPMTs of the pancreas from the files of the Diagnostic Pathology Division, National Cancer Center Hospital and National Cancer Center Hospital East. IPMTs of the 


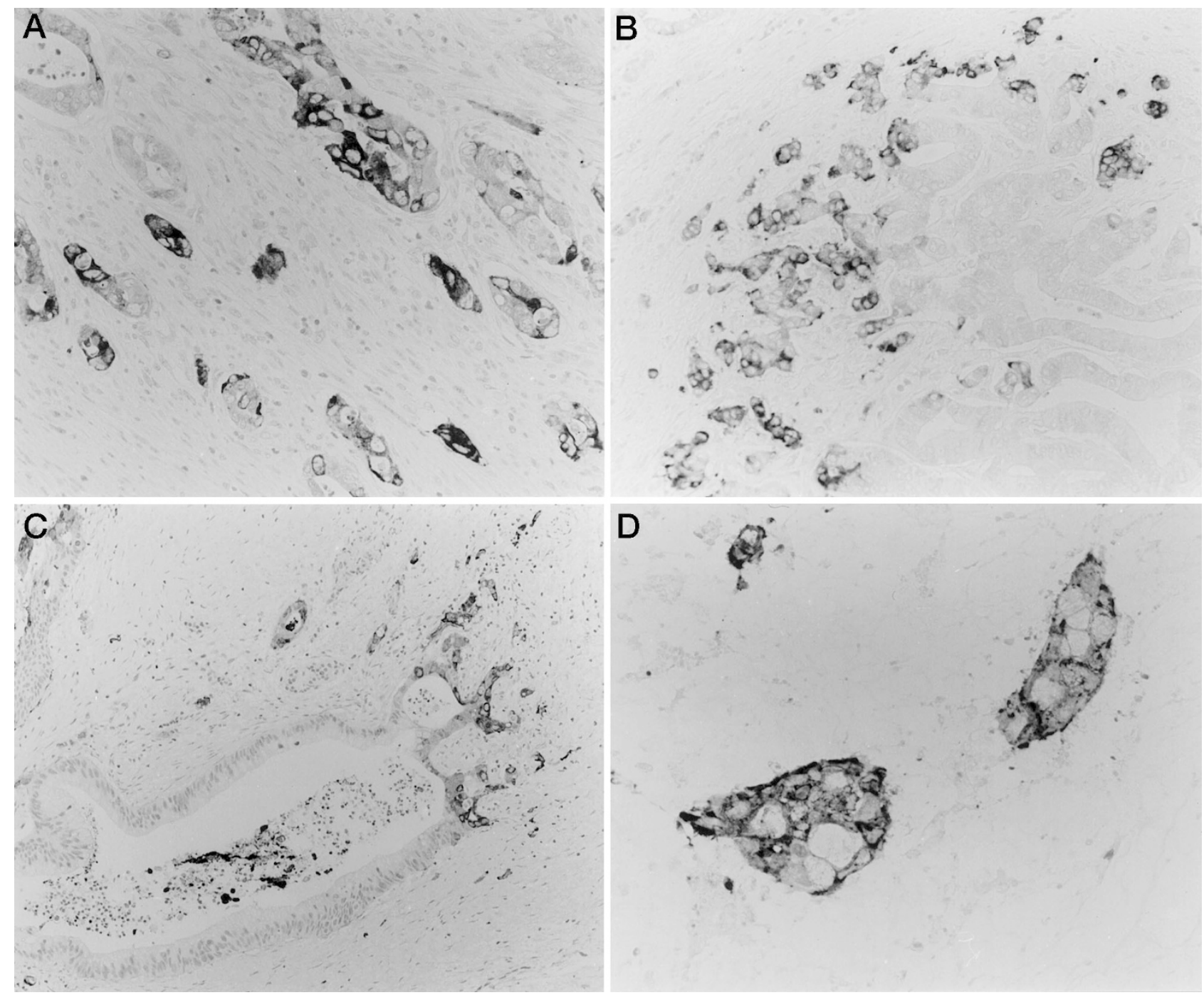

FIGURE 1. Immunohistochemical staining patterns of laminin-5- $\gamma$ - 2 chain in invasive carcinoma. A, The small tumor glands of components of invasive carcinoma show strong positivity. B, Part of a metastatic liver tumor. Positive staining is visible, predominantly in the periphery of the tumor nodules. C, Although the large tumor glands have stained weakly or not at all, the hornlike invasive fronts have stained positively. D, Tumor cells floating in the mucus lake showed positive staining in two cases.

pancreas are defined as epithelial proliferating tumors in the pancreatic duct that show varying degrees of cystic dilatation due to abundant mucus production. We classified the IPMTs into four categories: adenoma; adenocarcinoma without invasion; adenocarcinoma with minimal invasion; and adenocarcinoma with frank invasion recognizable by macroscopic observation. In "with minimal invasion" cases, there were only a small amount of invasive tumor glands or mucus lakes containing a few tumor cell clusters around the ectatic pancreatic ducts, which were not detected in macroscopical examination. These minimal invasion were usually observed within $5 \mathrm{~mm}$ from the ductal wall. We also added 30 invasive ductal adenocarcinomas (conventional type) of the pancreas in our study. Invasive ductal adenocarcinomas usually show solid and hard-tumor nodule with ill-defined margin. Many peripheral pancreatic ducts may be de- stroyed by the cancer invasion, but in some cases, intraductal tumor spread and intraductal papillary growth are observed within the area of tumor nodule. These findings were seen in 14 out of 30 cases. Our study cases therefore consisted of the following: intraductal papillary-mucinous adenoma ( $n=$ 8; Group A); intraductal papillary-mucinous adenocarcinoma without invasion ( $n=3$; Group $\mathrm{B}$ ), with minimal invasion ( $n=5$; Group C), or with macroscopic invasion ( $n=10$; Group D); and invasive ductal adenocarcinoma ( $n=30$; Group E). One case in Group D had liver metastasis at the time of surgery, and the tumor nodules were also resected.

\section{Laminin-5- $\gamma$-2-Chain Immunohistochemistry}

Mouse monoclonal antibody 1-97, specific to the laminin-5- $\gamma$-2 chain, was established in our laboratory as described by Ono et al. (15). We used this 
antibody for our immunohistochemical study. Formalin-fixed, paraffin-embedded sections were subjected to immunohistochemistry by the avidinbiotin-complex (ABC) method to characterize the tumor cells. Sections were deparaffinized with xylene, treated with $0.3 \%$ hydrogen peroxide in methanol, immersed in $10 \mathrm{~mm}$ citrate buffer $(\mathrm{pH} \mathrm{6.0)}$, heated to $120^{\circ} \mathrm{C}$ in an autoclave for 10 minutes, and then allowed to cool at room temperature for 30 minutes. The sections were then preincubated in $2 \%$ normal porcine serum in phosphate buffered saline, incubated with the purified monoclonal antibody 1-97 at room temperature overnight, washed with phosphate-buffered saline and incubated for 30 minutes with biotinylated horse anti-mouse immunoglobulin as the second antibody (Vector Laboratories, Burlingame, CA). Next, they were incubated for 30 minutes with avidin-biotinyl-peroxidase complex with a Vectastain ABC kit (Vector Laboratories) and subjected to the peroxidase reaction. This was followed by nuclear counterstaining with hematoxylin. Between each step, the slides were washed three times with phosphate buffered saline. The positive control was a section of formalin-fixed, paraffinembedded tissue of squamous cell carcinoma of the tongue, described elsewhere (15).

Immunoreactivity was evaluated as follows: - a few $(<3)$ positive areas; + , several (at least 3 ) positive areas; and ++ , many (at least 10 ) positive areas. In addition, the case with at least one large ( $>100$ cells) positive area was classified into ++ group.

\section{RESULTS}

No positivity of laminin-5- $\gamma$ - 2 chain was seen in the nonneoplastic ductal cells or in the acinic cells of the pancreas. One case in Group A and two in Group D showed focal or liner basement membrane stainings for laminin- 5 beneath the neoplastic epithelium. In nonneoplastic glands and ducts, basement membranes were not stained. The staining results were evaluated separately for the invasive and intraductal components of IPMTs.

\section{Invasive Components}

Laminin-5 staining was seen in the cytoplasms of the carcinoma cells and tended to be stronger in small tumor glands/solid nests than in large glands/solid nests (Fig. 1A). In the tumor nodules, staining was stronger in carcinoma cells at the periphery than in the central area (Fig. 1B). The tips of the glandular structure stained strongly (Fig. 1C).

In Group C, there were only a few positive areas in two cases. In Group D, several (+) positive areas were seen in 1 , and many $(++)$ positive areas were seen in 7 out of 10 cases (Table 1). Mucinous carcinoma-like invasion contained laminin-5-positive
TABLE 1. Laminin-5 $\gamma-2$ Chain Expression in Intraductal Papillary-Mucinous Adenocarcinoma with Minimal or Macroscopic Invasion

\begin{tabular}{cccc}
\hline $\begin{array}{c}\text { Case } \\
\text { No. }\end{array}$ & Noninvasive Component & Invasive Component \\
\hline & Adenocarcinoma with minimal invasion (Group C) & \\
1 & - & tub & - \\
2 & - & muc & - \\
3 & + & tub & - \\
4 & - & muc $>$ tub & - \\
5 & - & muc, tub & - \\
& Adenocarcinoma & with macroscopic invasion $(\mathrm{Group} \mathrm{D})$ & \\
1 & ++ & muc $>$ tub & - \\
2 & - & muc & $+{ }^{\mathrm{b}}$ \\
3 & + & muc, tub & ++ \\
4 & - & muc $>$ tub & - \\
5 & + & tub & ++ \\
6 & - & tub & ++ \\
7 & ++ & tub & ++ \\
8 & ++ & tub & ++ \\
9 & ++ & tub & ++ \\
10 & ++ & muc & $++{ }^{\mathrm{b}}$ \\
\hline
\end{tabular}

-, none or only a few ( $<$ three) positive areas; + , several $(\geq$ three) positive areas; ++ , many ( $\geq 10$ ) positive areas. In addition, the case with at least one large ( $>100$ cells) positive area was classified into ++ group.

a Histology of the invasive components: tub, tubular adenocarcinoma; muc, mucinous carcinoma-like invasion.

b Laminin-5-positive cells were seen in mucinous carcinoma-like invasive area.

floating-cell clusters in two cases (Table 1; Fig. 1D). Other positive cells were seen in the tubular adenocarcinomatous invasive area. The staining patterns of the invasive components of Group D and Group E were similar, especially in the areas of tubular invasion. In Group E, all 30 cases contained several $(+)$ or many $(++)$ laminin-5-positive cell foci.

Metastatic carcinoma cells of the liver were also stained by laminin-5 in one case (Group D). The tumor nodule was lobulated, and staining for laminin-5 was also strong at the epithelial-stromal interface, as in the primary lesion.

\section{Intraductal Components}

The intraductal components were also stained in some cases (Tables 1 and 2). In Groups A and B, there were only a few vague staining areas in one case. In contrast, in Group C, a few stainings were

TABLE 2. Immunohistochemical Stainings of Laminin-5 $\gamma-2$ Chain in Intraductal Components of Intraductal Papillary-Mucinous Tumors

\begin{tabular}{lllll}
\hline \multirow{2}{*}{ Group } & \multicolumn{3}{c}{ Staining } \\
\cline { 2 - 5 } & - & + & ++ & Total \\
\hline Adenoma (group A) & 8 & - & - & 8 \\
$\begin{array}{l}\text { Adenocarcinoma without invasion (group B) } \\
\begin{array}{c}\text { Adenocarcinoma with minimal invasion } \\
\quad \text { group C) }\end{array}\end{array}$ & 3 & - & - & 3 \\
$\begin{array}{c}\text { Adenocarcinoma with macroscopical invasion } \\
\text { (group D) }\end{array}$ & 3 & 2 & 5 & 10 \\
\hline
\end{tabular}

- , none or only a few ( $<$ three) positive areas; + , several $(\geq$ three) positive areas; ++ , many $(\geq 10)$ positive areas. In addition, the case with at least one large ( $>100$ cells) positive area was classified into ++ group. 
seen in two cases, and several positive stainings $(+)$ were seen in one case. Furthermore, several $(+)$ or many $(++)$ staining areas were detected in seven cases $(70 \%)$ in Group D (Table 2; Fig. 2). No apparent morphologic difference between laminin-5positive cells and negative cells was noticed. There was sometimes equivocal inflammation at the borders between the intraductal components and the stromal tissue. In these areas, laminin-5-positive cells were occasionally seen. Invasive cells were found adjacent to the intraductal laminin-5-positive cells. In Group E, we focused on the intraductal papillary components of 14 out of 30 tumors. Most of the intraductal papillary lesions did not stain for laminin-5, but a few foci stained positively in many cases, as in Group D (Fig. 3). It was difficult to compare the frequencies of occurrence of laminin5 -positive cells in the intraductal components of Groups D and E because it was difficult to distinguish the intraductal components from the invasive tumor glands in Group E.

\section{DISCUSSION}

Most IPMTs of the pancreas are noninvasive tumors, including adenoma and adenocarcinoma, which are cured by complete tumor resection. However, in some cases, extraductal tumor invasion is present. Intraductal papillary-mucinous carcinoma with extrapancreatic invasion and/or lymph node metastasis has a poor prognosis and should be treated as invasive ductal carcinoma in certain cases (6). Tumor extension may well be a determinant of prognosis in IPMTs, as in many other tumors.

All of our 30 invasive ductal adenocarcinomas of the pancreas contained laminin-5- $\gamma$-2-chain-positive cells on immunohistochemistry. Soini et al. (13) reported that intracytoplasmic positivity for laminin-5 was seen in $98 \%(41 / 42)$ of pancreatic adenocarcinomas. They also found that tumors forming solid nests or large glandular structures showed less laminin-5- $\gamma$-2-chain positivity than tumors with small invasive glandular structures or scattered individual invasive cells (14). The expression pattern of invasive carcinoma cells in our study was in accordance with the results of this and other reports $(9-11)$, and these results suggest that the expression of laminin-5 is associated with interaction between the epithelium and stroma.

In our study, one case in Group A and two in Group D showed focal or liner basement mem-

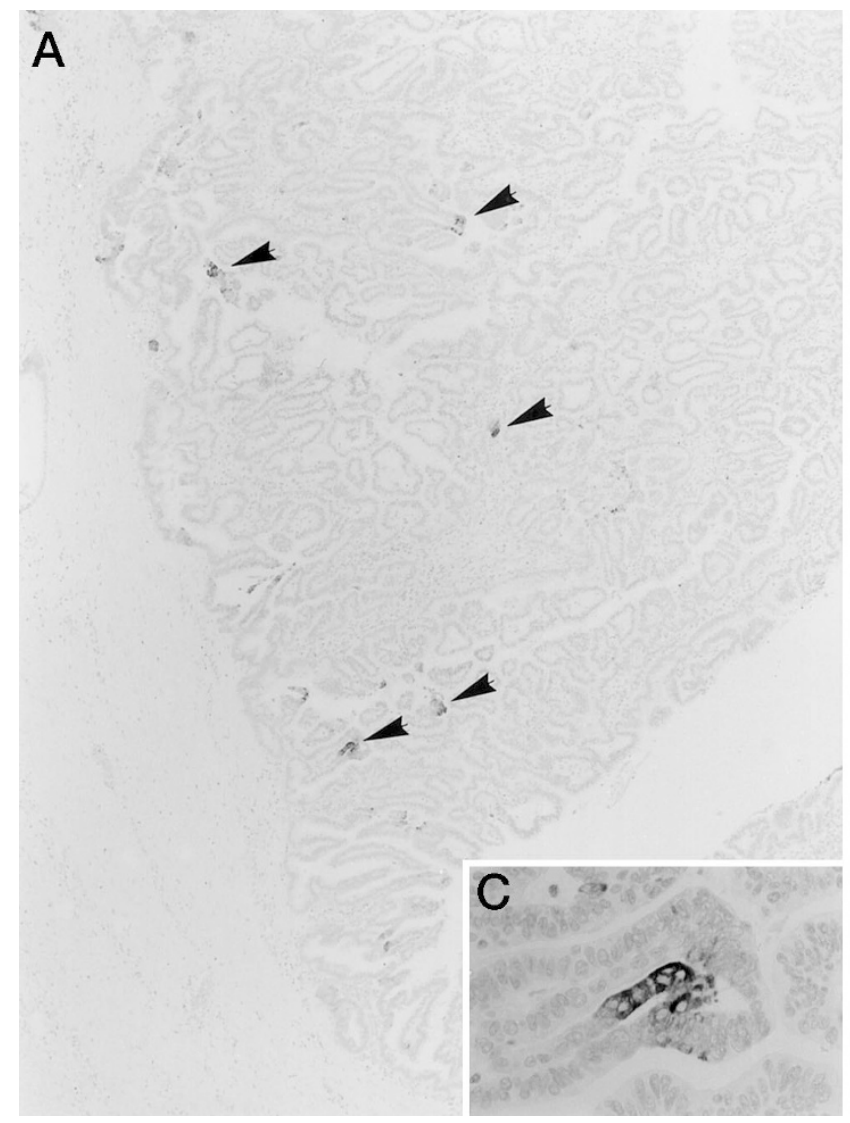

B

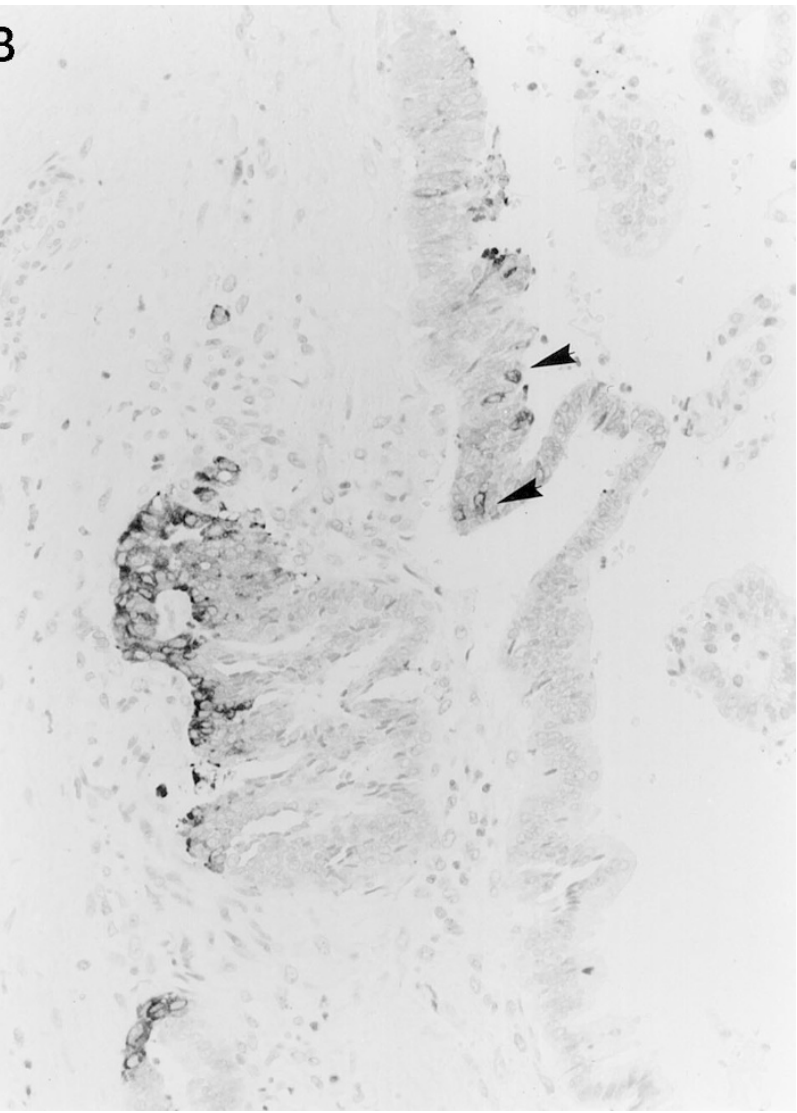

FIGURE 2. Laminin-5-gamma-2-chain immunohistochemical staining in intraductal papillary-mucinous tumors. A, There are several positive foci in the noninvasive tumor component. The cytoplasm of the intraductal tumor cells is stained (inset). B, The laminin-5-positive tumor gland is visible adjacent to an intraductal component that has stained positively for laminin-5. 


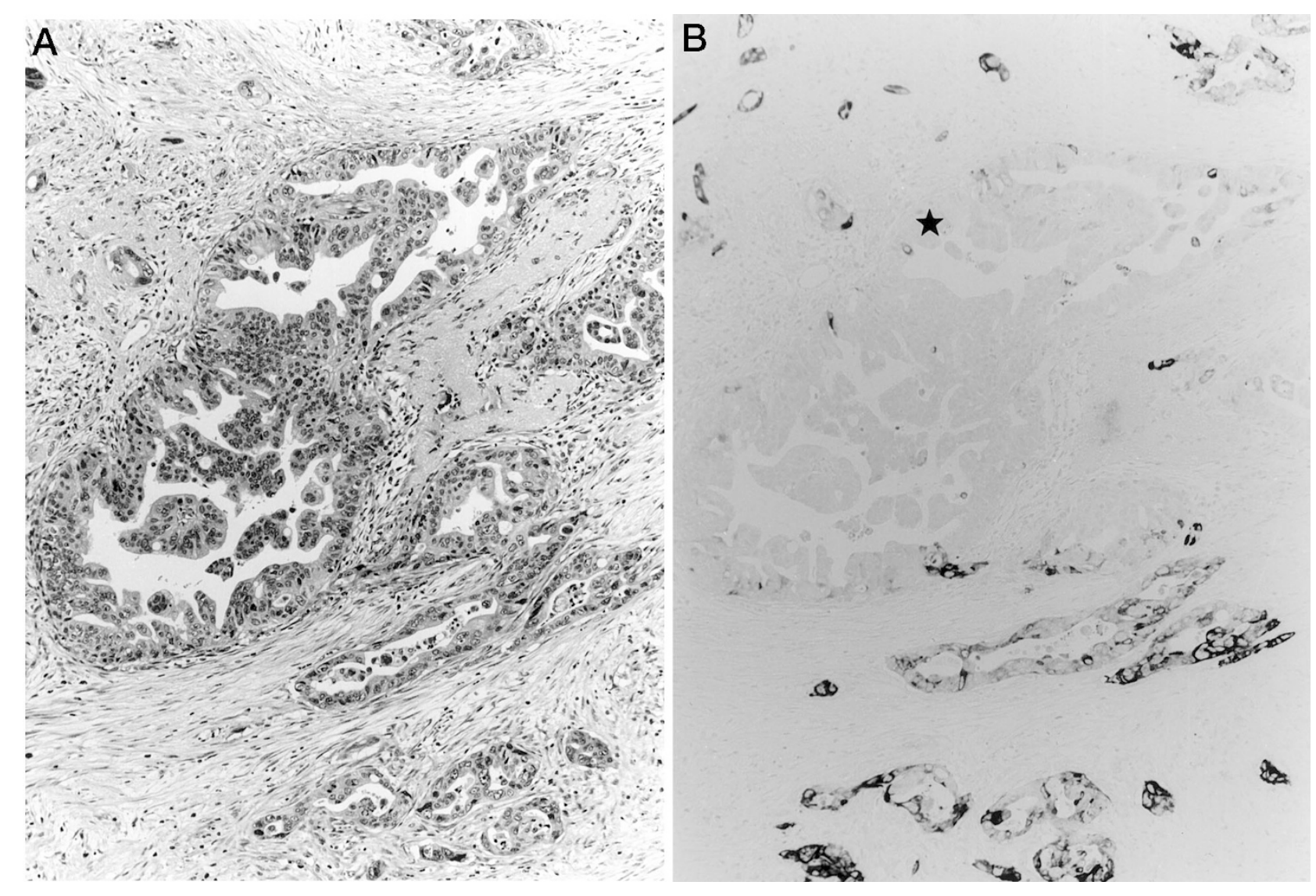

FIGURE 3. Laminin-5-gamma-2-chain immunohistochemical staining patterns in invasive ductal carcinoma with intraductal tumor components. There are a few positive stainings in the intraductal component. Most of the invasive tumor cells have stained positively. (Serial sections of the tumor: A, HE stain; B, laminin-5- $\gamma$-2 chain.)

brane stainings for laminin- 5 beneath the neoplastic epithelium. In nonneoplastic glands and ducts, basement membranes were not stained. Soini suggested that the concentration of laminin-5 in nonneoplastic basement membranes is so low that it cannot be detected by the antibody (13). Our results are in accordance with those of previous studies (9, $10,13)$.

In the World Health Organization classification of exocrine pancreatic tumors, intraductal papillarymucinous adenocarcinomas are divided into noninvasive and invasive types (papillary-mucinous carcinoma) according to "the absence or presence of neoplastic glandular structures invading the pancreatic tissue surrounding the involved ducts" (1). However, in some cases, it is difficult to judge whether or not there is invasion on the basis of morphology alone. Part of the mucus lake around the carcinomatous duct might be formed by physical rather than biological mechanisms (4), and sometimes it is difficult to differentiate tubular invasion from extension of the carcinoma into ductules. It is necessary to distinguish truly invasive cells with a biologically invasive nature from others. Our results indicate that immunohistochemical expression of laminin-5- $\gamma-2$ is a useful marker for detecting invasive tumor cells in IPMTs. Small tumor clusters, in particular, stained intensely. Floating tumor cells in mucus lakes also stained positively for laminin-5. If laminin-5 staining is used, more precise judgment of invasion than with hematoxylin-eosin stain alone in intraductal papillary-mucinous adenocarcinoma may be possible.

Recently, the biological characteristics of tumor progression from adenoma to noninvasive carcinoma and then to invasive carcinoma in IPMTs have been noted. Fujii et al. analyzed the patterns of allelic loss in IPMTs and reported that the histologically benign components of IPMT showed loss of $6 q$ and $9 p$, whereas the high-grade in situ carcinoma foci showed loss of $6 \mathrm{q}, 9 \mathrm{p}$, and $1 \mathrm{p}$ (17). Yonezawa et al. studied the expression of the apomucins MUC-1 and MUC-2 (18). A pattern of MUC-1 positivity and MUC-2 negativity was found in the invasive carcinomas, whereas MUC-1 negativity and MUC-2 positivity was frequently seen in IPMTs (18). Another recent study demonstrated that the invasive areas of intraductal papillary-mucinous adenocarcinoma were positive for expression of MUC-1 mucin but that the noninvasive areas were negative for expression of MUC-1 mucin (19). In 
our study, laminin-5-positive cells were usually seen in the invasive component of IPMTs, but they sometimes occurred in the intraductal component. The frequencies of occurrence of laminin-5-positive tumor cells in the intraductal component tended to increase with increasing tumor development grade, from adenoma to adenocarcinoma with macroscopic invasion. These findings suggest that the appearance of laminin-5-positive cells in the intraductal component might coincide with the acquisition of invasiveness by noninvasive tumor cells or might be connected to the invasive components. In other words, the noninvasive IPMTs containing laminin-5-positive cells might have developed into invasive carcinoma if pancreatic excision had not been performed.

In our immunohistochemical study of laminin-5$\gamma-2$ chain protein, we have demonstrated that the frequency of occurrence of laminin-5-positive cells in the intraductal component of IPMTs tends to increase with tumor development. And we can evaluate the invasion in IPMT more precisely by using laminin-5 immunohistochemistry as a useful adjunct to the morphological examination.

\section{REFERENCES}

1. Klöppel G, Solcia E, Longnecker DS, Capella C, Sobin LH, editors. Histological typing of tumors of the exocrine pancreas. (WHO international histological classification of tumors). 2nd ed. Tokyo: Springer-Verlag; 1996.

2. Japan Pancreas Society, editor. Classification of pancreatic cancer. 1st Engl. ed. Tokyo: Kanehara; 1996. p. 27-38.

3. Fukushima N, Mukai K. Pancreatic neoplasms with abundant mucus production: emphasis on intraductal papillarymucinous tumors and mucinous cystic tumors. Adv Anat Pathol 1999;6:65-77.

4. Fukushima N, Mukai K, Kanai Y, Hasebe T, Shimada K, Ozaki $\mathrm{H}$, et al. Intraductal papillary tumors and mucinous cystic tumors of the pancreas: clinicopathologic study of 38 cases. Hum Pathol 1997;28:1010-7.

5. Sessa F, Solcia E, Capella C, Bonato M, Scarpa A, Zamboni G, et al. Intraductal papillary-mucinous tumors represent a distinct group of pancreatic neoplasms: an investigation of tumor cell differentiation and K-ras, p53 and c-erbB-2 abnormalities in 26 patients. Virchows Archiv 1994;425:357-67.

6. Kimura W, Sasahira N, Yoshikawa T, Muto T, Makuuchi M. Duct-ectatic type of mucin producing tumor of the pancreas-new concept of pancreatic neoplasia. Hepatogastroenterology 1996;43:692-709.
7. Mizushima H, Miyagi Y, Kikkawa Y, Yamanaka N, Yasumitsu $\mathrm{H}$, Misugi K, et al. Differential expression of laminin-5/ladsin subunits in human tissue and cancer cell lines and their induction by tumor promoter and growth factors. J Biochem 1996;120:1196-202.

8. Kallunki P, Sainio K, Eddy R, Byers M, Kallunki T, Sariola H, et al. A truncated laminin chain homologous to the B2 chain: structure, spatial expression, and chromosomal assignment. J Cell Biol 1992;119:679-93.

9. Pyke C, Salo S, Ralfkiær E, Rømer J, Danø K, Tryggvason K. Laminin-5 is a marker of invading cancer cells in some human carcinomas and is coexpressed with the receptor for urokinase plasminogen activator in budding cancer cells in colon adenocarcinomas. Cancer Res 1995;55:4132-9.

10. Pyke C, Rømer J, Kallunki P, Lund L, Ralfkiær E, Danø K, et al. The $r 2$ chain of kalinin/laminin-5 is preferentially expressed in invading malignant cells in human cancers. Am J Pathol 1994;145:782-91.

11. Määttä M, Soini Y, Pääkko P, Salo S, Tryggvason K, AutioHarmainen $\mathrm{H}$. Expression of the laminin $r$-2 chain in different histological types of lung carcinoma. A study by immunohistochemistry and in situ hybridization. J Pathol 1999; 188:361-8.

12. Hao J, Yang Y, McDaniel KM, Dalkin BL, Cress AE, Nagle RB. Differential expression of laminin 5 (alpha3 bate3 gamma 2) by human malignant and normal prostate. Am J Pathol 1996; 149:1341-9.

13. Soini Y, Määttä M, Salo S, Tryggvason K, Autio-Harmainen $H$. Expression of the laminin $r$-2 chain in pancreatic adenocarcinoma. J Pathol 1996;180:290-4.

14. Tani T, Lumme A, Linnala A, Kivilaakso E, Kiviluoto T, Burgeson RE, et al. Pancreatic carcinomas deposit laminin-5 preferably adhere to laminin-5, and migrate on the newly deposited basement membrane. Am J Pathol 1997;151:1289302.

15. Ono Y, Nakanishi Y, Ino Y, Niki T, Yamada T, Yoshimura K, et al. Clinicopathologic significance of laminin-5 gamma2 chain expression in squamous cell carcinoma of the tongue: immunohistochemical analysis of 67 lesions. Cancer 1999; 85:2315-21.

16. Skyldberg B, Salo S, Eriksson E, Aspenblad U, Moberger B, Tryggvason $\mathrm{K}$, et al. Laminin-5 as a marker of invasiveness in cervical lesions. J Natl Cancer Inst 1999;91:1882-7.

17. Fujii H, Inagaki M, Kasai S, Miyokawa N, Tokusashi Y, Gabrielson $\mathrm{E}$, et al. Genetic progression and heterogeneity in intraductal papillary-mucinous neoplasms of the pancreas. Am J Pathol 1997;151:1447-54.

18. Yonezawa S. Application of immunohistochemistry for diagnosis of neoplasms: mucin antigens expression and biological behavior of neoplasms. Acta Histochem Cytochem 1994; 27:561-6.

19. Yonezawa S, Taira M, Osako M, Kubo M, Tanaka S, Sakoda K, et al. MUC-1 mucin expression in invasive areas of intraductal papillary mucinous tumors of the pancreas. Pathol Int 1998;48:319-22. 